\title{
Modèle numérique de clapage
}

\section{Phase de chute}

\author{
Isabelle Farout-Fréson* — Emmanuel Lefrançois* \\ Gouri Dhatt* — Philippe Sergent**
}

* Laboratoire ROBERVAL UMR CNRS 6253

Laboratoire d'Hydraulique Numérique, BP 20529, F-60205 Compiègne cedex

\{isabelle.freson,emmanuel.lefrancois,gouri.dhatt\}@utc.fr

** Centre d'Etudes Techniques Maritimes et Fluviales

2 boulevard Gambetta BP 60039

F-60321 Compiègne cedex

philippe.sergent@equipement.gouv.fr

RÉSUMÉ. Nous nous intéressons ici aux mélanges fluide incompressible/particules solides fluidisées. Le modèle proposé est basé sur l'écriture moyennée des équations hydrodynamiques biphasiques associée à une équation de transport de sédiments munie d'une vitesse numérique de chute $w_{s f}$ spécifiquement adaptée au cas des rejets de dragage par clapage. La stratégie de résolution entre l'hydrodynamique et le transport est réalisée en considérant une variation de la densité du mélange de façon explicite afin d'obtenir un modèle couplé de manière séquentielle. Calibré sur la phase de chute de trois campagnes expérimentales de clapage en canal, le modèle donne de très bons résultats sur près de vingt essais de rejets sableux, sablo vaseux, vaseux avec et sans courant ambiant (Villaret et al., 1997 ; Boutin 1999).

ABSTRACT. We are interested here in the mixture composed of incompressible fluid and a certain mass of fluidised solid. The proposed model is based on the averaged form of the hydrodynamic biphasic equations, associated with a sediment transport equation with a specific numerical settling velocity $w_{s f}$ adapted for the dumped dredged material case. Both models (hydrodynamics and transport) are coupled considering the variation of density with a forward scheme. Calibrated on the convective descent on three experimental campaigns in canal of dumping of dredged materials, the model gives a very good agreement of convective descent with almost twenty experiments for materials 100\% sand, 100\% silt or mixture sand/silt without or with a horizontally ambient current (Villaret et al., 1997; Boutin 1999).

MOTS-CLÉS: mélange, fluide incompressible, densité variable, systèmes couplés, hydrodynamique, transport sédimentaire, non linéaire, vitesse de chute, clapage, rejets de dragage.

KEYWORDS: mixture, incompressible fluid, variable density, coupled system, hydrodynamics, sediment transport, non linear, settling velocity, dumping, dredged sediment.

DOI:10.3166/REMN.16.965-988 (c) 2007 Lavoisier, Paris 


\section{Intérêts de l'étude}

Les ports maritimes et les voies navigables sont soumis régulièrement à des envasements qui rendent la navigation difficile voire dangereuse. Afin de faciliter les trafics maritimes et fluviaux, chaque port doit procéder à des dragages d'entretien. Ces dragages nécessaires représentent par an et en France un volume de rejets allant de 40 à 50 millions de mètres cubes. Ces rejets sont composés essentiellement de sables, de boues, de vases mais aussi de polluants d'origine variée (hydrocarbures, plastiques, etc.). Les matériaux de dragage constitués de sédiments grossiers sont parfois utilisés pour la construction de routes, la réhabilitation de plages en cours d'érosion ou encore l'enrichissement de terres agricoles mais le plus souvent ces matériaux se composent d'un mélange de sédiments fins et n'ont d'autres alternatives que d'être transférés ailleurs, dans une zone maritime qui empêcherait les matériaux de venir se redéposer à leur point initial et où l'impact sur l'environnement serait mineur. La recherche de sites d'immersions, pour minimiser cet impact sur l'environnement, s'avère ainsi être une opération des plus complexes. Elle requiert une connaissance précise de l'évolution de la bathymétrie suite aux dépôts, de l'interaction du rejet avec les courants environnants, des propriétés diffusives des différents matériaux constituant le rejet... Un outil de calcul numérique s'avère aujourd'hui comme un moyen utile voire nécessaire pour améliorer la connaissance des phénomènes impliqués et faciliter la décision de retenir ou non un site donné.

Du point de vue réglementaire, les conditions d'immersion des rejets de dragage sont fixées au niveau international par la convention d'Oslo. La prise en compte de ces dispositions en 1996 amène les autorités françaises à missionner un groupe de travail sur les opérations de dragage et leur impact sur l'environnement pour faire le point sur les connaissances scientifiques en matière d'impact des opérations de dragage et d'immersion, établir la demande en besoin de recherche et proposer un programme de recherche pour combler les lacunes identifiées. De cette mission naît le projet « Liteau clapage » dont l'objectif est de recenser les outils de prédiction disponibles pour étudier l'impact des rejets de dragage sur le littoral. Des essais physiques sont alors réalisés de 1997 à 2001 au sein du LNHE-EDF à Chatou (France) pour des rejets $100 \%$ sable (Burel et Villaret, 2000 ; Villaret et al., 1997), sablo vaseux et $100 \%$ vase (Boutin, 1999) et une synthèse des modèles de rejet de dragage montre un déficit en modèle de rejet dans des sites d'immersion de faible profondeur (inférieure à 100 mètres) avec courant ambiant qui représentent la quasi-totalité des sites d'immersion français. Le travail, présenté ici, tente de palier cette lacune en modélisant dans un premier temps la phase de chute de ces rejets de dragage par clapage, issus de chalands à clapets.

S'appuyant sur les observations de clapage en nature de Bokuniewicz et al., (1978) ou in situ de (Johnson et Fong, 1995 ; Truitt, 1988 ; Villaret et al., 1997 et Boutin, 1999) les rejets de dragage par clapage en eau peu profonde suivent les trois phases caractéristiques suivantes (illustration figure 1) :

- une phase de chute très rapide pendant laquelle le matériau chute dans la colonne d'eau sous l'influence dominante de la gravité : qu'il soit constitué de sédiments 
cohésifs ou non, le rejet se comporte comme un « jet dense » jusqu'à une centaine de mètres environ;

- une phase d'effondrement dynamique à l'arrivée sur le fond ou dans des couches de densité proche de celle du rejet à cet instant un courant de densité se forme pour s'étendre de façon purement radiale autour du point d'impact;

- une phase de transport dépôt sur le fond régie par le courant de densité : le rejet est entraîné tout en se mélangeant aux couches inférieures de la colonne d'eau et les sédiments finissent par se déposer dès que l'énergie de l'effondrement est dissipée.

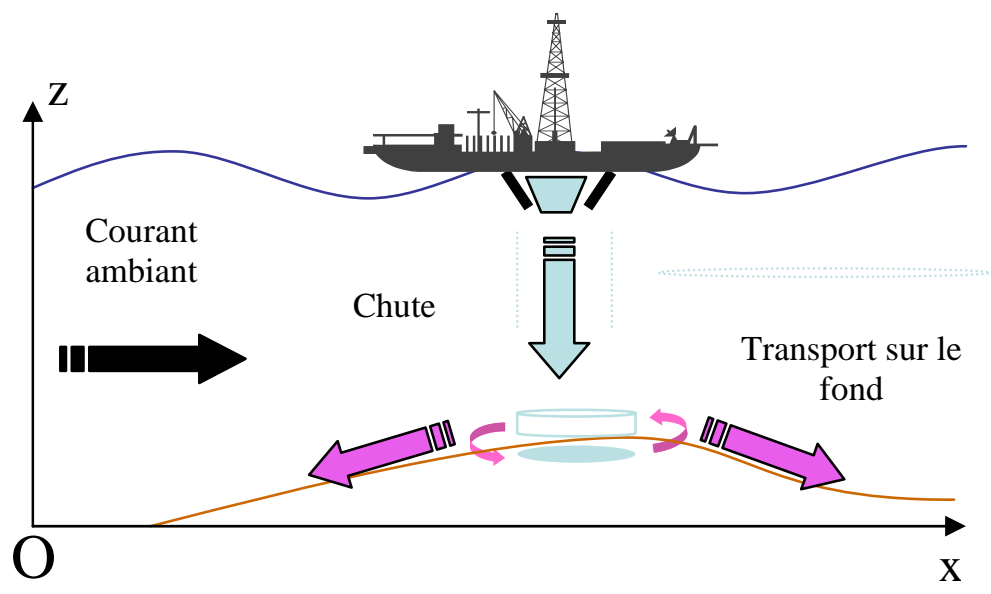

Figure 1. Représentation d'un rejet de dragage par clapage

Les sites de clapage français ont une profondeur relativement faible au regard des sites américains ou canadiens et n'excèdent pas la centaine de mètres ; de plus ils sont assujettis à de forts courants maritimes atteignant parfois plus d'un mètre par seconde sur le fond (sites de Dunkerque Ouest Nord et Ouest Sud, sites de Rouen le Kannick et intermédiaire) et jusqu'à un mètre vingt par seconde en surface (site de Dunkerque Est). Les principales modélisations existantes (Krischnappan, 1976 ; Drapeau et al., 1992 ; Johnson et Fong, 1995) sont adaptées aux sites très profonds (canadiens et américains) pour lesquels la phase de chute se termine par une phase de sédimentation sans impact massif sur le fond. Elles s'appuient essentiellement sur des approximations d'ordre semi-empirique ou géométrique mais ne parviennent pas à simuler correctement la chute des rejets dans les sites peu profonds à fort courant ambiant. Or une bonne approximation des dépôts nécessite une bonne simulation des phases en amont et en particulier de la phase de chute. Nous portons donc ici une attention toute particulière aux rejets de dragage sur les sites de clapage français dont la profondeur n'excède pas soixante mètres et pour lesquels les courants de fond ou de surface peuvent être importants. 
L'analyse de Roland Boutin sur de tels rejets montre que les vitesses de chute observées en milieu naturel, sont très élevées et comprises entre 25 et $280 \mathrm{~cm} / \mathrm{s}$ et ne peuvent donc être modélisées par les lois classiques de vitesse de chute sédimentaire exprimées par bon nombre d'auteurs (Richardson et Zaki, 1954 ; Tambo et al., 1979 ; Migniot, 1989; Van Leussen, 1993 entre autres). Pour les cas qu'il présente, Gordon (1974) confirme également que la vitesse de chute du nuage d'une valeur moyenne de $40 \mathrm{~cm} / \mathrm{s}$, est nettement supérieure à la vitesse de chute des particules individuelles qui le composent, elle-même estimée à $0,5 \mathrm{~cm} / \mathrm{s}$.

L'objet de ce travail est donc de présenter un modèle numérique destiné à simuler le phénomène de clapage pour des rejets $100 \%$ sable, sablo vaseux voire $100 \%$ vase avec ou sans courant ambiant. Deux approches sont possibles. L'approche biphasique est l'approche la plus naturelle, elle permet de décrire les caractéristiques propres à chacune des phases du mélange en résolvant les équations de l'hydrodynamique sédimentaire pour l'eau et le sédiment fluidisé. Reportant chacune de ces phases à sa part de masse au sein du mélange, l'approche bi-espèce qui découle directement de l'approche biphasique après moyenne «massique», permet de décrire non plus l'évolution moyenne de chaque phase mais l'évolution du mélange en tant que tel (Farout-Fréson, 2004). De manière générale, l'approche biphasique permet d'extraire des données qualitatives propres à chacune des phases considérées, au détriment du temps global de calcul du fait de la double résolution des équations de Navier-Stokes. Le modèle bi-espèce est quant à lui plus rapide puisqu'il considère une grandeur moyennée (une seule résolution de Navier-Stokes) mais ne permet cependant pas d'obtenir des informations relatives à chacune des phases. C'est cette deuxième approche bi-espèce que nous exposons ici.

Une première section décrit l'ensemble des variables utiles selon l'approche biphasique. Une seconde section pose le modèle mathématique associé à l'approche bi-espèce. Nous présentons ensuite le modèle aux éléments finis et le couplage réalisé entre l'hydrodynamique et le transport. Le modèle bi-espèce 2D est enfin appliqué au cas des rejets de dragage par clapage avec la formulation d'une nouvelle vitesse de chute numérique spécifique. Les résultats numériques sont ensuite comparés aux essais physiques expérimentaux du LNHE-EDF (Chatou, France), confirmant la bonne tenue du modèle pour la phase de chute de tous types de rejets qu'ils soient $100 \%$ sable, sablo vaseux, $100 \%$ vase avec ou sans courant ambiant.

\section{Définition des variables selon les approches biphasique et bi-espèce}

Afin de faciliter la compréhension du modèle de calcul présenté ci-après, nous définissons ici un ensemble de variables permettant de décrire un mélange eausédiments selon les approches biphasique et bi-espèce. Considérons un volume de contrôle V de mélange constitué d'une composante fluide (l'eau de mer) et d'une composante sédimentaire (sédiments supposés fluidisés) dont les densités réelles respectives $\rho_{f_{0}}, \rho_{s_{0}}$ sont des paramètres fixes. Les proportions de masse solide $m_{s}$ et 
fluide $m_{f}$ par rapport au volume $\mathrm{V}$ sont définies par leur densité apparente $\rho_{f}, \rho_{s}$ et volume apparent $V_{f}, V_{s}$ selon :

$$
\rho_{s}=\frac{m_{s}}{V}, \quad \rho_{f}=\frac{m_{f}}{V} \quad \text { avec } \quad m_{s}=V_{s} \rho_{s_{0}} \quad m_{f}=V_{f} \rho_{f_{0}}
$$

Les fractions volumiques de chaque phase, correspondent à la part de volume qu'occupe chaque phase dans le volume de contrôle :

$$
\varphi_{s}=\varphi=\frac{V_{s}}{V}, \quad \varphi_{f}=\frac{V_{f}}{V}, \text { avec } \varphi_{f}+\varphi=1
$$

La densité apparente, est alors directement exprimée à partir de la densité réelle de chaque composant du mélange par :

$$
\rho_{s}=\varphi \rho_{s_{0}}, \quad \rho_{f}=\varphi_{f} \rho_{f_{0}}=(1-\varphi) \rho_{f_{0}}
$$

La densité moyenne du mélange est représentée par la somme des densités partielles :

$$
\rho=\rho_{s}+\rho_{f}=\varphi \rho_{s_{0}}+(1-\varphi) \rho_{f_{0}}
$$

Les fractions massiques des parts de masse sédimentaire et fluide au sein $\mathrm{du}$ mélange sont :

$$
C=C_{s}=\frac{\rho_{s}}{\rho} \quad C_{f}=1-C=\frac{\rho_{f}}{\rho}
$$

Utilisant [3] et [4] nous obtenons la fraction massique en fonction de la fraction volumique :

$$
C=\frac{\varphi \rho_{s_{0}}}{\varphi \rho_{s_{0}}+(1-\varphi) \rho_{f_{0}}} \text { et } \varphi=\frac{\rho_{f_{0}} C}{\rho_{f_{0}} C+(1-C) \rho_{s_{0}}}
$$

Le modèle bi-espèce correspond à un modèle biphasique moyenné massiquement. On déduit ainsi des précédentes équations, la relation pour la densité moyenne $\rho \mathrm{du}$ mélange en fonction de $\mathrm{C}$ :

$$
\rho=\frac{\rho_{f_{0}} \rho_{s_{0}}}{(1-C) \rho_{s_{0}}+C \rho_{f_{0}}}
$$


La vitesse du mélange est quant à elle définie par pondération massique de la vitesse sédimentaire $\vec{u}_{s}$ et fluide $\vec{u}_{f}$ :

$$
\vec{u}^{(m)}=C \vec{u}_{s}+(1-C) \vec{u}_{f}
$$

Enfin, une répartition des pressions est établie par composant et la pression moyenne massique du mélange est définie par la somme des deux selon :

$$
p_{s}=C p \text { et } p_{f}=(1-C) p \text { avec } p=p_{s}+p_{f}
$$

\section{Modèles mathématiques}

Nous présentons respectivement dans cette section, le modèle biphasique puis le modèle bi-espèce qui en résulte.

\subsection{Modèle biphasique}

Une description selon une approche biphasique de l'écoulement du mélange utilise une approche continue pour chaque phase. Celles-ci peuvent alors être représentées par les équations de conservation de la masse et de la quantité de mouvement de chacune d'elles. Tous les effets thermodynamiques sont ici négligés.

$$
\begin{aligned}
& \left\{\begin{array}{l}
\frac{\partial \rho_{i}}{\partial t}+\operatorname{div}\left(\rho_{i} \vec{u}_{i}\right)=0 \\
\frac{\partial \rho_{i} \vec{u}_{i}}{\partial t}+\operatorname{div}\left(\rho_{i} \vec{u}_{i} \otimes \vec{u}_{i}-\overline{\bar{\sigma}}_{i}\right)+\vec{F}_{i f}-\rho_{i} \vec{g}=\overrightarrow{0}
\end{array} \quad \text { avec } i=\left\{\begin{array}{l}
s: \text { sédiment } \\
f: \text { fluide }
\end{array}\right.\right. \\
& \overline{\bar{\sigma}}_{i}=-p_{i} \overline{\bar{I}}+2 \mu_{i} \overline{\bar{\varepsilon}}_{i} \text { (Anderson et Jackson 1967) }
\end{aligned}
$$

Les variables $\rho_{i}, \vec{u}_{i}, \overline{\bar{\sigma}}_{i}, \overline{\bar{\varepsilon}}_{i}$ définissent resp. la densité moyenne, le vecteur vitesse et les tenseurs des contraintes et déformations de la phase $i$. Les termes $\vec{F}_{s f}$ et $\vec{F}_{f s}$ représentent les forces d'interaction entre la phase fluide et la phase solide. Les termes $p_{i}$ et $\mu_{i}$ définissent la pression et la viscosité associées à la phase $i$.

\subsection{Modèle bi-espèce}

Le modèle bi-espèce est un dérivé moyenné du modèle biphasique. Les deux phases sont toujours présentes mais ne sont plus explicitement caractérisées de 
manière individuelle. Les grandeurs permettent ici de déterminer l'évolution moyenne d'un mélange de deux phases et non l'évolution de chaque phase.

Les équations hydrodynamiques moyennes du modèle sont obtenues par la somme respective des équations de conservation de la quantité de mouvement et de la masse des phases fluide et solide du modèle biphasique.

Equation de continuité : la sommation des équations de conservation des masses fluides et sédimentaires, associée à l'équation de la fraction massique sédimentaire conduit à :

$$
\frac{\partial \rho}{\partial t}+\operatorname{div}\left(\rho\left(C \vec{u}_{s}+(1-C) \vec{u}_{f}\right)\right)=0
$$

De l'équation de la vitesse moyenne massique nous obtenons l'équation moyennée de conservation de la masse du mélange :

$$
\frac{\partial \rho}{\partial t}+\operatorname{div}\left(\rho \vec{u}^{(m)}\right)=0
$$

C'est une formulation pénalisée (pseudocompressibilité) qui est ici retenue pour conduire à la relation (Hubert et Dhatt, 1983) :

$$
\operatorname{div}\left(\rho \vec{u}^{(m)}\right)=\frac{p}{\lambda}
$$

Le coefficient de pénalité considéré est $\lambda=10^{8}$. Cette approche permet notamment une condensation du système algébrique sur le champ de pression pour n'aboutir qu'à des inconnues en vitesses.

Equations de conservation de la quantité de mouvement: la somme des équations de conservation de la quantité de mouvement de chaque phase aboutit à :

$$
\frac{\partial\left(\rho_{s} \vec{u}_{s}+\rho_{f} \vec{u}_{f}\right)}{\partial t}+\vec{\nabla} \cdot\left(\rho_{s} \vec{u}_{s} \otimes \vec{u}_{s}+\rho_{f} \vec{u}_{f} \otimes \vec{u}_{f}\right)-\vec{\nabla} \cdot\left(\bar{\sigma}_{s}+\bar{\sigma}_{f}\right)+\vec{F}_{s f}+\vec{F}_{f s}-\left(\rho_{s}+\rho_{f}\right) \vec{g}=\overrightarrow{0}
$$

Les forces d'interactions opposées définies à l'aide d'un coefficient d'interaction $k_{s f}$ s'annulent :

$$
\vec{F}_{s f}+\vec{F}_{f s}=k_{s f}\left(\vec{u}_{s}-\vec{u}_{f}\right)+k_{s f}\left(\vec{u}_{f}-\vec{u}_{s}\right)=\overrightarrow{0}
$$


La répartition des contraintes est estimée selon la moyenne massique :

$$
\overline{\bar{\sigma}}_{s}=C \overline{\bar{\sigma}} \quad \text { et } \bar{\sigma}_{f}=(1-C) \overline{\bar{\sigma}} \text { où } \overline{\bar{\sigma}}=\bar{\sigma}_{s}+\overline{\bar{\sigma}}_{f}
$$

Une répartition massique des viscosités est établie par composant et la viscosité moyenne du mélange est définie par la somme des deux selon :

$$
\mu_{s}=C \mu^{(m)} \text { et } \mu_{f}=(1-C) \mu^{(m)} \text { avec } \mu=\mu_{s}+\mu_{f}
$$

La contribution de la gravité sur le mélange est :

$$
\left(\rho_{s}+\rho_{f}\right) \vec{g}=\rho \vec{g}
$$

Nous obtenons par ailleurs :

$$
\frac{\partial}{\partial t}\left(\rho_{s} \vec{u}_{s}+\rho_{f} \vec{u}_{f}\right)=\frac{\partial}{\partial t}\left(\rho C \vec{u}_{s}+\rho(1-C) \vec{u}_{f}\right)=\frac{\partial}{\partial t}\left(\rho \vec{u}^{(m)}\right)
$$

Pour la somme des produits tensoriels de chaque phase nous avons :

$$
\vec{u}_{s}=\vec{u}^{(m)}+(1-C)\left(\vec{u}_{s}-\vec{u}_{f}\right) \quad \text { et } \quad \vec{u}_{f}=\vec{u}^{(m)}-C\left(\vec{u}_{s}-\vec{u}_{f}\right)
$$

Ceci nous amène à :

$$
\rho_{s} \vec{u}_{s} \otimes \vec{u}_{s}+\rho_{f} \vec{u}_{f} \otimes \vec{u}_{f}=\rho\left[\vec{u}^{(m)} \otimes \vec{u}^{(m)}+\left[C(1-C)\left(\vec{u}_{s}-\vec{u}_{f}\right) \otimes\left(\vec{u}_{s}-\vec{u}_{f}\right)\right]\right] .
$$

Les termes croisés $\vec{u}^{(m)} \otimes\left(\vec{u}_{s}-\vec{u}_{f}\right)$ s'éliminent naturellement et par analogie au tenseur des contraintes de Reynolds de la turbulence, nous écrivons $2 \mu_{s f} \overline{\dot{\varepsilon}}=-\rho C(1-C)\left(\vec{u}_{s}-\vec{u}_{f}\right) \otimes\left(\vec{u}_{s}-\vec{u}_{f}\right)$.

Nous obtenons les équations moyennées de conservation de la quantité de mouvement du modèle bi-espèce :

$$
\frac{\partial \rho \vec{u}^{(m)}}{\partial t}+\operatorname{div}\left(\rho \vec{u}^{(m)} \otimes \vec{u}^{(m)}+p \overline{1}-2\left(\mu^{(m)}+\mu_{s f}\right) \overline{\dot{\varepsilon}}\right)-\rho \vec{g}=\overrightarrow{0}
$$

En notant désormais la vitesse en moyenne massique $\vec{u}^{(m)}=\vec{u}$, et la viscosité globale du mélange $\mu=\mu^{(m)}+\mu_{s f}$, [20] s'écrit finalement : 


$$
\frac{\partial \rho \vec{u}}{\partial t}+\operatorname{div}(\rho \vec{u} \otimes \vec{u}+p \overline{1}-2 \mu \overline{\bar{\varepsilon}})-\rho \vec{g}=\overrightarrow{0}
$$

Equation de transport: l'évolution de $\mathrm{C}$ est quant à elle entièrement déterminée par l'équation de conservation de la masse sédimentaire :

$$
\frac{\partial \rho_{s}}{\partial t}+\operatorname{div}\left(\rho_{s} \vec{u}_{s}\right)=0 \Leftrightarrow \frac{\partial(\rho C)}{\partial t}+\operatorname{div}\left((\rho C) \vec{u}_{s}\right)=0
$$

Comme dans la théorie de la turbulence, la vitesse sédimentaire est ici décomposée en une vitesse moyenne $\vec{u}^{(m)}$ et une vitesse de fluctuation $\overrightarrow{\tilde{u}}_{s}$, qui gère tous les effets secondaires dus aux interactions entre les particules sédimentaires et le fluide.

$$
\vec{u}_{s}=\vec{u}^{(m)}+\overrightarrow{\tilde{u}}_{s} \text { où } \overrightarrow{\tilde{u}}_{s}=\vec{u}_{s f}-k_{\text {diff }} \vec{\nabla} C
$$

Ces effets sont essentiellement des effets d'entraînement d'une phase par rapport à l'autre, qui se manifestent principalement par convection (terme $\vec{u}_{s f}$ ) et diffusion (terme $-k_{\text {diff }} \vec{\nabla} C$ ). Insérée dans l'équation de conservation de la masse sédimentaire nous obtenons finalement la loi de conservation de $\mathrm{C}$ par convection et diffusion :

$$
\frac{\partial(\rho C)}{\partial t}+\operatorname{div}\left(\rho C\left(\left(\vec{u}^{(m)}+\vec{u}_{s f}\right)-k_{\text {diff }} \vec{\nabla} C\right)\right)=0
$$

Nous obtenons ainsi dans ce modèle une partie purement hydrodynamique pour le calcul des champs de vitesse et de la pression et une partie transport de la fraction sédimentaire du mélange. La discrétisation par la méthode des éléments finis requiert l'écriture sous forme variationnelle de ces deux parties. Ainsi pour toute fonction de pondération $\delta \vec{u}, \delta p, \delta C$ appartenant à l'ensemble des solutions admissibles qui satisfont les conditions aux limites du problème bi-espèce, l'application de la méthode des résidus pondérés aboutit aux formulations intégrales suivantes :

$W_{N S}$ Conservation de la quantité de mouvement

$$
\begin{aligned}
& \int_{\Omega} \delta \vec{u} \cdot \frac{\partial \rho \vec{u}}{\partial t} d S+\int_{\Omega} \delta \vec{u} \vec{\nabla}(\rho \vec{u} \otimes \vec{u}) d S-\int_{\Omega} \overline{\overline{\delta \varepsilon}}: p \overline{\overline{1}} d S+\int_{\Omega} \overline{\overline{\delta \varepsilon}}: \overline{\bar{\tau}} d S+\int_{\partial \Omega} \delta \vec{u} \cdot(p \overline{\overline{1}}-\overline{\bar{\tau}}) \vec{n} d l \\
& -\int_{\Omega} \delta \vec{u} \rho \vec{g} d S=0 \quad \text { avec } \overline{\bar{\tau}}=2 \mu \bar{\varepsilon} \quad \text { et } \quad \stackrel{\bar{\varepsilon}}{=}=\frac{1}{2}\left(\vec{\nabla} \vec{u}+\vec{\nabla}^{T} \vec{u}\right) .
\end{aligned}
$$


$W_{M}$ Conservation de la masse du mélange avec pénalité sur la pression

$$
W_{M}=\int_{\Omega} \delta p \operatorname{div}(\rho \vec{u}) d S-\int_{\Omega} \delta p \frac{p}{\lambda} d S=0
$$

$W_{C}$ Conservation de la masse sédimentaire

$$
\begin{aligned}
& \int_{\Omega} \delta C \frac{\partial(\rho C)}{\partial t} d S-\int_{\Omega} \vec{\nabla}(\delta C) \cdot\left(\vec{u}+\vec{u}_{s f}\right) \rho C d S+\int_{\Omega} \vec{\nabla}(\delta C) \cdot \rho C k_{d i f f} \vec{\nabla} C d S \\
& +\int_{\partial \Omega} \delta C \rho C\left(\left(\vec{u}+\vec{u}_{s f}\right)-k_{d i f f} \vec{\nabla} C\right) \cdot \vec{n} d l=0
\end{aligned}
$$

Cette formulation est complétée d'un ensemble de conditions aux limites sur tout le contour et de conditions initiales sur tout le domaine. Elles seront explicitées dans la section « Applications ».

Les deux sections suivantes décrivent respectivement l'hydrodynamique et le transport du modèle bi-espèce ainsi que la méthode de couplage permettant d'assurer une mise à jour régulière des données communes et complémentaires des deux parties.

\section{Modèle éléments finis}

La discrétisation spatiale du modèle est basée sur la méthode des éléments finis (Dhatt et al., 2005). L'élément considéré est un quadrilatère à quatre nœuds de type Q4 basé sur une approximation bilinéaire du champ de vitesse et de la concentration :

$$
\begin{array}{ll}
u(\xi, \eta)=\sum_{i=1}^{4} N_{i}(\xi, \eta) u_{i}, & C(\xi, \eta)=\sum_{i=1}^{4} \sum_{i} N_{i}(\xi, \eta) C_{i} \\
u(\xi, \eta) w(\xi, \eta)=\sum_{i=1}^{4} N_{i}(\xi, \eta) u_{i} \cdot w_{i}, & \rho(\xi, \eta) u(\xi, \eta)=\sum_{i=1}^{4} N_{i}(\xi, \eta) \rho_{i} \cdot u_{i}
\end{array}
$$

où $u_{i}, w_{i}, C_{i}$ et $\rho_{i}$ sont resp. les valeurs nodales des deux composantes de la vitesse du mélange, de la fraction massique et de la densité moyenne.

Pour des raisons de stabilité numérique (Dhatt et al., 2005), la pression est considérée constante sur l'élément. Le calcul des matrices et vecteurs élémentaires est obtenu par intégration numérique sur quatre points de Gauss. Pour le détail des matrices élémentaires se référer à (Farout-Fréson, 2004). A l'issue de la phase d'assemblage, nous obtenons ainsi respectivement pour le modèle « hydrodynamique » et le modèle de « transport », les systèmes discrets : 
Partie hydrodynamique : $\left[M_{U}\right]\{\dot{U}\}+\left[K_{U}(U)\right]\{U\}=\left\{F_{U}\right\}$

Partie Transport : $\quad\left[M_{C}\right]\{\dot{C}\}=-\left[K_{C}\right]\{C\}$

Une condensation sur le champ de pression permet de réduire le modèle « hydrodynamique » aux seules variables de vitesse.

La discrétisation en temps pour les deux modèles est basée sur un schéma de type Euler implicite qui assure une stabilité inconditionnelle pour le modèle linéaire de transport et conditionnelle pour le modèle "hydrodynamique » non linéaire (condition difficile à déterminer). A ceci, s'ajoute la non linéarité due aux couplages successifs des deux modèles. La stabilité sera donc assurée pour un pas de temps n'entraînant l'apparition d'aucune instabilité numérique. Les termes non linéaires sont traités par une technique classique de linéarisation de Newton-Raphson, méthode permettant d'assurer une convergence rapide à proximité de la solution.

\section{Modèle de couplage}

La stratégie de couplage entre l'hydrodynamique et le transport est basée sur le déroulement successif (modèle séquentiel) des deux modèles tel qu'il est illustré figure 2.

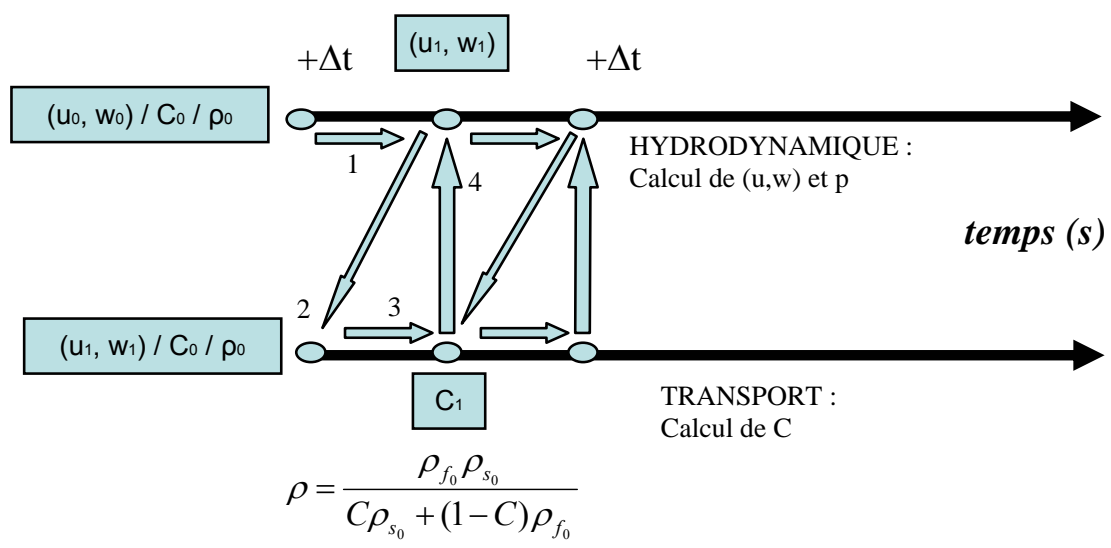

Figure 2. Couplage des modèles d'hydrodynamique et de transport

La lecture d'un cycle est la suivante :

- le modèle d'hydrodynamique calcule une mise à jour du champ de vitesse en considérant une densité constante sur l'intervalle de temps ;

- ce dernier transmet au modèle de transport le nouveau champ de vitesse ; 
- le modèle de transport évalue le «transport» de la concentration à partir $d u$ champ de vitesses mis à jour puis en déduit le champ $\rho_{t+\Delta t}$;

- transmission au modèle d'hydrodynamique du nouveau champ $\rho_{t+\Delta t}$;

- retour étape 1 pour un nouveau cycle...

Pour des raisons évidentes de simplifications, aucun sous-cyclage n'est introduit, à savoir l'exécution successive de $\mathrm{N}$ pas de calcul pour un modèle donné entre deux exécutions de l'autre modèle. Les deux modèles sont donc incrémentés du même intervalle de temps $\Delta \mathrm{t}$. L'approche par sous-cyclage est intéressante pour des cas de figure où les pas de temps caractéristiques sont d'un ordre de grandeur différent entre les deux parties et permet d'éviter ainsi de pénaliser un des deux codes par le plus restrictif des deux pas de temps. Elle requiert cependant l'ajout d'une phase itérative supplémentaire lors d'un cycle complet de calcul afin d'éviter les soucis de stabilité numérique qui résulteraient d'un trop important décalage entre les deux modèles (Piperno et Farhat, 2000).

L'algorithme général de résolution par couplage des modèles d'hydrodynamique et de transport est donné dans l'algorithme ci-après :

\section{Algorithme général de résolution}

Calcul des matrices et vecteurs globaux constants

Conditions initiales : $\left\{U_{t}\right\}=\{U\}\left\{C_{t}\right\}=\{C\}\left\{\rho_{t}\right\}=\{\rho\}$

Boucle sur le pas de temps

- Hydrodynamique

Actualisation la matrice masse $\left[M_{U}\right]$

Calcul de la matrice tangente $\left[K_{T}(U)\right]$

Calcul du résidu $\{R\}=\Delta t\left(\left\{F_{U}\right\}-\left[K_{U}(U)\{U\}\right]\right)-\left[M_{U}\right]\left(U-U_{t}\right)$

Calcul de l'incrément de solution : $\left\{\left[K_{T}\right]\{\Delta U\}\right\}=\{R\}$

Mise à jour de la solution : $\{U\}=\{U\}+\{\Delta U\}$

Test de convergence : $\|n\|=\operatorname{Max}\left|\frac{\Delta U}{U}\right|<10^{-8}$

- Transport

Calcul de : $\left[M_{C}\right]$ et $\left[K_{C}(U)\right]$

Calcul de la solution : $\left(\left[M_{C}\right]+\Delta t\left(\left[K_{C}\right]\right)\right)\{\Delta C\}=-\Delta t\left(\left[K_{C}\right]\right)\left\{C_{t}\right\}$ 
Mise à jour : $\{C\}=\left\{C_{t}\right\}+\{\Delta C\}$ et $\left\{\rho_{t+\Delta t}\right\}=\{\rho(C)\}$

Retour boucle sur le temps

\section{Application}

\subsection{Recalage du modèle bi-espèce}

Le modèle présenté a requis une phase de validation et de recalage adaptée spécifiquement au cas du clapage en eau peu profonde pour la vitesse de chute sédimentaire (loi de type suspension). Cette dernière a été rendue possible à partir des mesures physiques du LNHE-EDF pour des rejets $100 \%$ sable avec et sans courant ambiant (mesures référencées B8, V6 et V12 : Farout-Fréson 2004).

Elle est donnée en fonction des caractéristiques initiales du rejet en particulier le diamètre moyen des sédiments par l'intermédiaire de la vitesse de chute des particules sédimentaires $w_{s}$, et la concentration volumique initiale. La vitesse de chute numérique ainsi définie permet d'obtenir une phase de chute cohérente pour tous les types de rejets qu'ils soient $100 \%$ sable, sablo vaseux ou $100 \%$ vase avec et sans courant ambiant. Par ailleurs, $w_{s f}$ représente environ un dixième de la vitesse de chute moyenne du nuage $w_{c}$ et dix fois la vitesse de chute des sédiments $w_{s}$ du mélange. Nous avons donc adopté pour tous les rejets une formulation de type suspension utilisée en sédimentologie classique mais adaptée ici au phénomène du clapage selon :

$$
w_{s f}=-\alpha_{s f} C^{v} w_{s} \text { avec } w_{s}=f\left(D_{50}\right)
$$

où :

$-C^{v}(g / l):$ Concentration initiale du rejet;

$-\alpha_{s f} \quad:$ Coefficient numérique propre aux simulations de clapage ;

: Rejets $100 \%$ sable, $100 \%$ vase ou sablo vaseux $\alpha_{s f}=0.0225$;

- $D_{50} \quad$ : Diamètre moyen des grains de sédiment composant le mélange ;

- $w_{s} \quad:$ Vitesse sédimentaire d'une particule moyenne du mélange ;

$-f\left(D_{50}\right)\left\{\begin{array}{l}: \text { si rejets } 100 \% \text { sable } \Rightarrow \text { Formulation de Van Rijn (Van Rijn 1993); } \\ : \text { si rejets sablo-vaseux } \Rightarrow w_{s}=\varphi_{s, \text { sable }} w_{s, \text { sable }}+\left(1-\varphi_{s, \text { sable }}\right) w_{s, \text { vase }} \\ : \text { si rejets } 100 \% \text { vase } \Rightarrow \text { Formulation de Stokes (Migniot 1989). }\end{array}\right.$

L'obtention de cette loi unique résulte d'une campagne de recalage numérique des codes de calcul sur l'ensemble des données fournies par la campagne expérimentale du LNHE-EDF. 


\subsection{Spécificité des mélanges sablo vaseux et calcul de la vitesse sédimentaire $w_{s}$}

Le modèle bi-espèce 2D est un modèle numérique dont les équations prennent initialement en compte deux espèces (espèce fluide et espèce sédimentaire) au sein d'un même mélange. Les rejets sablo vaseux ont la particularité d'être un mélange fluide/sédiment dont la partie sédimentaire est elle-même un mélange de deux types de sédiment. Pour que les équations puissent traiter ce type de rejet, il est nécessaire de calculer les nouvelles propriétés de cette partie sédimentaire (Farout-Fréson et al., 2005). Pour cela, nous nous plaçons dans l'espace qui nous intéresse, c'est-à-dire le volume de sédiment, et nous calculons la fraction volumique correspondant au sédiment de type sable $\varphi_{s, \text { sable }}$ et la fraction volumique correspondant au sédiment de type vase $\varphi_{s, \text { vase }}$, soit :

$$
\varphi_{s, \text { vase }}=\frac{V_{\text {vase }}}{V_{\text {solide }}} \text { et } \varphi_{s, \text { sable }}=\frac{V_{\text {sable }}}{V_{\text {solide }}}=1-\varphi_{s, \text { vase }}
$$

Pour la vase, la vitesse sédimentaire est calculée par la formule de Stokes. Pour le sable, elle est donnée par la formulation de Van Rijn, et la vitesse sédimentaire moyenne attribuée à l'ensemble des sédiments composant le mélange sablo vaseux est calculée par pondération convexe selon la formulation ci-après :

$$
w_{s}=\varphi_{\text {sable }} w_{s, \text { sable }}+\left(1-\varphi_{\text {sable }}\right) w_{s, \text { vase }}
$$

\subsection{Domaine d'étude, conditions initiales et conditions aux limites}

Un dispositif mis au point par Boutin a permis d'entreprendre des essais de clapage in situ dans le canal $\mathrm{n}^{\circ} 5 \mathrm{du}$ Laboratoire national d'hydraulique et environnement de EDF à l'échelle $1 / 25^{\mathrm{e}}$. Il a été utilisé par Boutin pour une dizaine de rejets par clapage $100 \%$ vase dès 1996 , puis par Villaret pour tous les clapages $100 \%$ sable et sablo vaseux. Le cas d'application choisi est issu des essais de clapages in situ de Villaret et référencés V2 à V20 (Farout-Fréson, 2004). Il a été retenu en raison des très nombreuses mesures qui lui sont associées.

Il s'agit d'un canal rectangulaire fermé avec une frontière amont pour axe de symétrie, le fond et le côté aval représentent les limites du domaine hors couche limite et sont donc assimilés à des parois glissantes (l'objectif étant ici de conserver des tailles de maillage raisonnables) mais implique donc la non prise en compte des couches limites. La surface libre qui n'est pas modélisée ici, est aussi assimilée à une paroi glissante. Ces simplifications gardent une justification tant que l'analyse porte sur la seule phase de chute. 
Pour le système hydrodynamique, nous imposons sur toute la frontière de normale $\vec{n}$, une composante normale nulle ainsi qu'une composante tangentielle de contrainte visqueuse nulle :

$$
\vec{u} . \vec{n}=u_{n}=0 \text { sur } \quad \partial \Omega \quad \tau_{t}=0 \text { sur } \partial \Omega
$$

Pour le transport sédimentaire, nous considérons les flux de sédiments nuls à la surface et au fond :

$$
\left(w-w_{s f}\right) C-k_{\text {diff }} C \frac{\partial C}{\partial z}=0 \text { sur } \quad \partial \Omega_{\text {surface }} \cup \partial \Omega_{\text {fond }}
$$

Ces conditions aux limites permettent d'annuler tous les termes de contours des formes variationnelles [25], [26] et [27]. Dans ce modèle, aucun phénomène d'érosion/dépôt sur le fond n'est pris en considération et aucun échange ne s'effectue avec la surface.

Les conditions initiales sont définies par la vitesse du courant ambiant horizontal $\vec{U}_{a}$ et la concentration massique initiale de sédiments clappés $C_{0}$ :

$$
\vec{u}(t=0)=\vec{U}_{a}=\left(u_{a}, 0\right) \text { et } C(t=0)=C_{0} .
$$

\subsection{Résultats}

\section{Essais sans courant ambiant}

La symétrie d'un rejet sans courant ambiant ayant été observée expérimentalement et numériquement, les simulations sont effectuées sur un demi-domaine comportant un demi-rejet pour les clapages sans courant ambiant (figure 3). Le domaine de simulation est un rectangle de hauteur unité pour une longueur de quatre mètre. Une concentration initiale homogène non nulle $C_{0}$ est initialement répartie sur un rectangle de $0,17 \mathrm{~m}$ de hauteur sur $0,055 \mathrm{~m}$ de largeur.

La position d'un rejet $Y$ s'effectue par sa position la plus basse avec un seuil de fraction massique délibérément fixé à $C \leq C_{0} / 30$. Le rayon du rejet $\mathrm{R}$ correspond au cours de la chute à la largeur la plus importante du rejet avec un seuil de fraction massique délibérément fixé à $C \leq C_{0} / 100$.

Une analyse de sensibilité de ces seuils n'a pas permis de montrer de forte dépendance sur les résultats. La position initiale du rejet est donc fixée à $Y=0,83 \mathrm{~m}$ et le rayon initial équivaut $R=0,055 \mathrm{~m}$. 


\section{Essais avec courant ambiant}

Ce phénomène de symétrie n'étant plus de rigueur pour les rejets dont le milieu récepteur subit les influences d'un courant ambiant, nous simulons un demi-domaine comportant l'intégralité du rejet pour les clapages avec courant (figure 3) soit une concentration homogène initiale non nulle $C_{0}$ répartie sur un rectangle de $0,17 \mathrm{~m}$ de hauteur sur $0,11 \mathrm{~m}$ de largeur. Le diamètre du rejet $\mathrm{D}$ est ici estimé par la largeur maximum effective de la masse sédimentaire en chute avec le même seuil de fraction massique que précédemment. La position initiale du rejet est toujours fixée à $\mathrm{Y}=0,83 \mathrm{~m}$ et le diamètre initial équivaut à la largeur totale du rectangle sédimentaire initial soit $\mathrm{D}=0,11 \mathrm{~m}$.

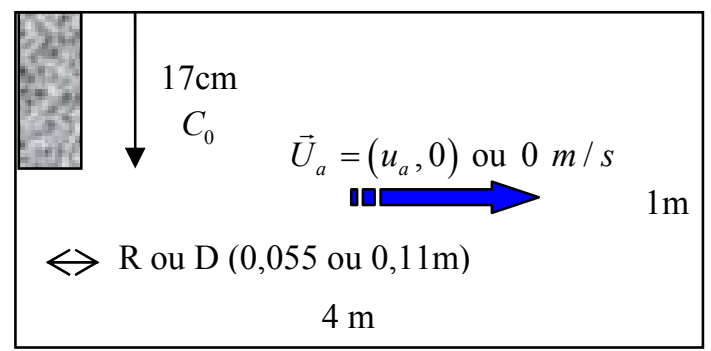

Figure 3. Domaine de simulation des essais avec et sans courant ambiant

Pas de temps : les résultats concernant la phase de chute des essais expérimentaux sont donnés pour un laps de temps minimum toutes les $0,13 \mathrm{~s}$. Ainsi, pour permettre une comparaison effective de nos résultats numériques avec ceux des essais, nous avons choisi un pas de temps garantissant un maximum de données comparables, et toutes nos simulations utilisent le pas de temps suivant : $\Delta t=0,1 \mathrm{~s}$

Maillage : le pas d'espace vertical $\Delta z=0,0333 m$, soit 31 nœuds sur la hauteur, est une valeur moyenne des différents écarts expérimentaux observés pour la position. Le pas d'espace horizontal, quant à lui $\Delta x=0,0392 \mathrm{~m}, 101$ nœuds sur $4 \mathrm{~m}$ de longueur, permet un temps de calcul relativement raisonnable pour un modèle écrit en MATLAB (logiciel de calcul scientifique, Mathworks(C) : $1 \mathrm{mn} 45$ par pas de temps soit moins de 3 heures de temps de calcul pour 10 secondes de simulation sur Pentium III cadencé à $1 \mathrm{GHz}$. Une analyse de sensibilité de l'influence de la longueur du domaine (1, 2, 3 et $4 \mathrm{~m}$ ) a de plus permis de déterminer une longueur suffisante pour ne pas influencer les résultats.

$$
\text { Données: } \rho_{f_{0}}=1000 \mathrm{~kg} / \mathrm{m}^{3} \quad \rho_{s_{0}}=2650 \mathrm{~kg} / \mathrm{m}^{3} \mathrm{~g}=9.81 \mathrm{~m} / \mathrm{s}^{2}
$$

Viscosité numérique du mélange massique : $\mu=10^{-3} \mathrm{~kg} / \mathrm{ms}$

Cette valeur de viscosité est comparable en ordre de grandeur aux valeurs observées en environnement turbulent et permet de plus d'assurer la stabilité du 
schéma. Bien qu'aucun modèle de turbulence ne soit utilisé, l'approche proposée ici est donc analogue à un modèle à viscosité turbulente constante.

Les figures 4 et 5 montrent l'adéquation des résultats numériques par rapport à l'expérimentation en ce qui concerne les positions $\mathrm{Y}(\mathrm{m})$ et rayons $\mathrm{R}(\mathrm{m})$ ou diamètres $\mathrm{D}(\mathrm{m})$ du rejet au cours du temps. La figure 4 présente le cas de 45 litres de rejet $60 \%$ sable (40\% vase) de concentration volumique initiale $350 \mathrm{~g} / \mathrm{l}$, dans un canal au repos. La figure du haut montre les positions du rejet (position la plus basse) en fonction du temps au cours de la phase de chute pour l'essai expérimental concerné V3 et la simulation effectuée par notre modèle bi-espèce 2D. La comparaison des données numériques et expérimentales montre la bonne représentation du modèle. La figure du bas donne le rayon du rejet (largeur maximum) en fonction du temps au cours de la chute ; là encore l'adéquation des résultats est intéressante. On retrouve sur la figure 5, le même type de représentation que sur la figure 6 avec 45 litres de rejet $20 \%$ sable ( $80 \%$ vase) pour une concentration volumique initiale de $350 \mathrm{~g} / 1$ et un courant ambiant au sein du canal de $10 \mathrm{~cm} / \mathrm{s}$ et nous retrouvons encore une bonne adéquation des résultats numériques et expérimentaux.

Le tableau 1 compare les résultats des modèles de clapage de Krishnappan, STFATE (utilisé par Ifremer Brest) et bi-espèce 2D, pour la phase de chute d'un rejet de 60 litres $100 \%$ sable de diamètre $90 \mu \mathrm{m}$ avec une concentration volumique initiale de $450 \mathrm{~g} / \mathrm{l}$ et sans courant ambiant (Farout-Fréson 2004: essai physique référencé V11). Nous constatons que le modèle empirique de Krishnappan donne une approximation relativement correcte de la phase de chute avec un diamètre maximum à l'impact de $62 \mathrm{~cm}$ contre $64 \mathrm{~cm}$ pour les mesures.
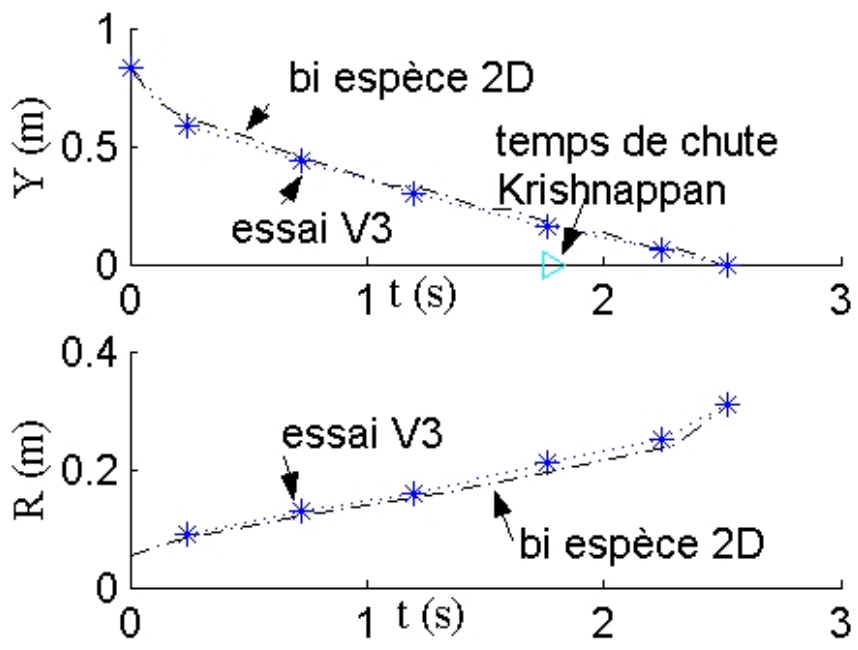

Figure 4. Positions et diamètres au cours de la chute d'un rejet sablo vaseux à $60 \%$ de sable de concentration volumique $350 \mathrm{~g} / \mathrm{l}$ sans courant ambiant 

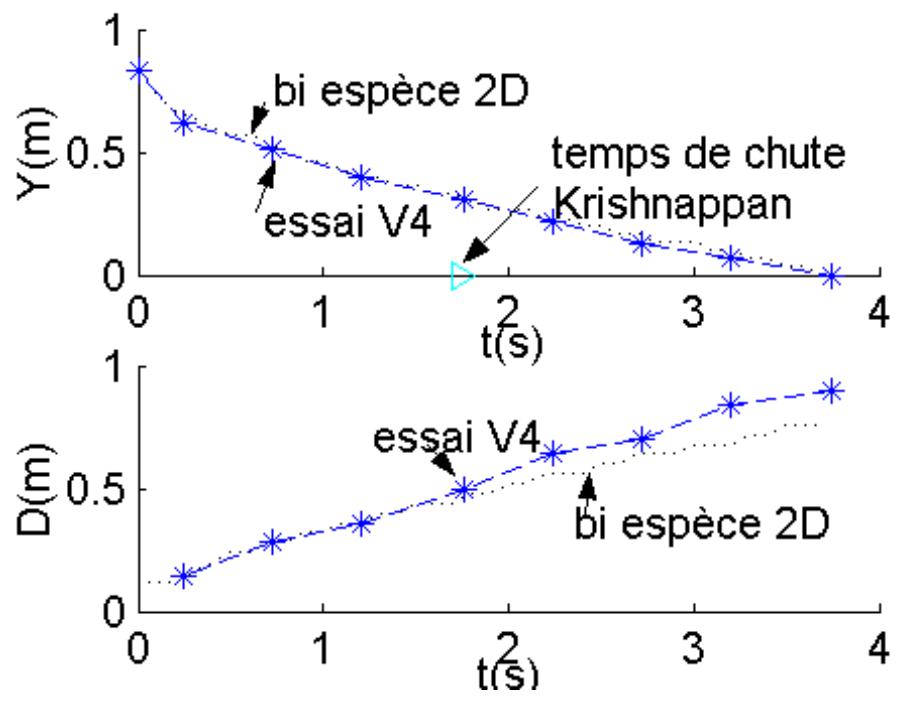

Figure 5. Positions et diamètres d'un rejet sablo vaseux à $20 \%$ de sable de concentration volumique $350 \mathrm{~g} / \mathrm{l}$ dans un courant ambiant de $10 \mathrm{~cm} / \mathrm{s}$

\begin{tabular}{|l|c|c|c|c|}
\hline Essai $100 \%$ sable $90 \mu \mathrm{m}$ & Mesures & Krishnappan & STFATE & Bi-espèce 2D \\
\hline $\mathrm{Ua}=0 \mathrm{~cm} / \mathrm{s} 601,450 \mathrm{~g} / 1$ & EDF 1997 & 1976 & Ifremer & UTC 2004 \\
\hline Instant ouverture (s) & 0,00 & 0,00 & 0,00 & 0,00 \\
\hline Position rejet Y(m) & 0,83 & 0,83 & 0,44 & 0,83 \\
\hline Diamètre D(m) & 0,15 & 0,10 & 0,30 & 0,11 \\
\hline Temps (s) & 0,24 & 0,14 & 0,33 & 0,20 \\
\hline $\mathrm{Y}(\mathrm{m})$ & 0,56 & 0,60 & 0,32 & 0,50 \\
\hline Diamètre D(m) & 0,18 & 0,24 & 0,33 & 0,24 \\
\hline Temps (s) & 0,72 & 0,67 & 0,66 & 0,70 \\
\hline Y(m) & 0,18 & 0,20 & 0,05 & 0,13 \\
\hline Diamètre D(m) & 0,45 & 0,48 & 0,38 & 0,48 \\
\hline Temps (s) & 0,95 & 1,06 & 1,05 & 1,00 \\
\hline Y(m) & 0,00 & 0,00 & $(-) 0,28$ & 0,00 \\
\hline Diamètre D(m) & 0,64 & 0,62 & 0,44 & 0,64 \\
\hline Wchute moy (m/s) & 0,87 & 0,78 & 0,69 & 0,83 \\
\hline
\end{tabular}

Tableau 1. Comparaison des chutes d'un rejet $100 \%$ sable sans courant ambiant

La vitesse moyenne de chute du rejet avec ce modèle est de $78 \mathrm{~cm} / \mathrm{s}$ et sous estime largement la vitesse moyenne de chute de l'essai physique qui est de $87 \mathrm{~cm} / \mathrm{s}$. Le modèle américain STFATE (Short Term FATE of dredged material) testé par 
l'Ifremer (Brest) n'est pas du tout adapté aux sites à faibles profondeurs ; il ne permet pas d'obtenir l'impact sur le fond et sous estime très largement le diamètre et la vitesse moyenne de chute. Le modèle bi-espèce $2 \mathrm{D}$ apporte des résultats très satisfaisants avec une estimation du diamètre à l'impact en adéquation avec l'expérimentation et une vitesse moyenne de chute de $83 \mathrm{~cm} / \mathrm{s}$ contre $87 \mathrm{~cm} / \mathrm{s}$ pour l'essai.

De même, le tableau 2 compare les chutes d'un rejet $100 \%$ sable (diamètre $160 \mu \mathrm{m} 60$ litres avec une concentration volumique initiale de $450 \mathrm{~g} / \mathrm{l}$ ) avec courant ambiant.

Le modèle empirique de Krishnappan ne prenant pas du tout en considération le courant ambiant, montre ici ses limites avec une vitesse moyenne de chute totalement surestimée par rapport à l'essai physique. STFATE permet d'obtenir une vitesse moyenne de chute meilleure que celle de Krishnappan mais avec une surestimation de prés de $15 \mathrm{~cm} / \mathrm{s}$. Le modèle bi-espèce $2 \mathrm{D}$ apporte là encore des résultats très satisfaisants puisque l'estimation du diamètre à l'impact et la vitesse moyenne de chute est en parfaite adéquation avec l'essai physique.

Le tableau 3 compare les temps de chute du modèle bi-espèce 2D aux temps de chute de dix sept essais physiques différents. Dans la colonne de gauche, sont exprimées les caractéristiques du rejet expérimental : volume du rejet, concentration volumique initiale de sédiment, pourcentage de sable pour les rejets sablo vaseux, taille moyen des grains de sable et de vase, intensité du courant ambiant.

\begin{tabular}{|l|c|c|c|c|}
\hline Essai100\% sable 160 $\mu \mathrm{m}$ & Mesures & Krishnappan & STFATE & Bi-espèce 2D \\
\hline Ua=20cm/s 601, 450 g/1 & EDF 1997 & 1976 & Ifremer & UTC 2004 \\
\hline Instant ouverture (s) & 0,00 & 0,00 & 0,00 & 0,00 \\
Position rejet Y(m) & 0,83 & 0,83 & 0,44 & 0,83 \\
Diamètre D(m) & 0,15 & 0,10 & 0,30 & 0,11 \\
\hline Temps (s) & 0,24 & 0,14 & 0,25 & 0,20 \\
Y(m) & 0,55 & 0,60 & 0,36 & 0,70 \\
Diamètre D(m) & 0,15 & 0,24 & 0,33 & 0,20 \\
\hline Temps (s) & 0,72 & 0,67 & 0,50 & 0,70 \\
Y(m) & 0,23 & 0,20 & 0,19 & 0,27 \\
Diamètre D(m) & 0,29 & 0,48 & 0,36 & 0,36 \\
\hline Temps (s) & 1,28 & 1,06 & 0,75 & 1,30 \\
Y(m) & 0,12 & 0,00 & $(-) 0,02$ & 0,17 \\
Diamètre D(m) & 0,47 & 0,62 & 0,40 & 0,52 \\
\hline Temps (s) & 1,60 & 1,06 & 1,07 & 1,60 \\
Y(m) & 0,00 & 0,00 & $(-) 0,28$ & 0,00 \\
Diamètre D(m) & 0,60 & 0,62 & 0,45 & 0,60 \\
\hline Wchute moy (m/s) & 0,52 & 0,78 & 0,67 & 0,52 \\
\hline
\end{tabular}

Tableau 2. Comparaison des chutes d'un rejet $100 \%$ sable avec courant ambiant 


\begin{tabular}{|c|c|c|c|c|}
\hline $100 \%$ sable 60 litres $450 \mathrm{~g} / \mathrm{l}$ & $\mathrm{T}(\mathrm{s})$ chute & $\mathrm{T}(\mathrm{s})$ chute & Erreur & Erreur \\
\hline Influence du courant & essai & bi-espèce $2 \mathrm{D}$ & $\Delta \mathrm{t}(\mathrm{s})$ & $\mathbf{\%}$ \\
\hline $\mathrm{D} 50=90 \mu \mathrm{m} \quad \mathrm{Ua}=0 \mathrm{~m} / \mathrm{s}$ & 0,95 & 1 & 0,05 & $\mathbf{5 , 2 6 \%}$ \\
\hline $\mathrm{D} 50=160 \mu \mathrm{m} \quad \mathrm{Ua}=0 \mathrm{~m} / \mathrm{s}$ & 0,72 & 0,7 & 0,02 & $\mathbf{2 , 7 7 \%}$ \\
\hline $\mathrm{D} 50=90 \mu \mathrm{m} \quad \mathrm{Ua}=0,1 \mathrm{~m} / \mathrm{s}$ & 1,54 & 1,6 & 0,06 & $\mathbf{3 , 8 9 \%}$ \\
\hline $\mathrm{D} 50=90 \mu \mathrm{m} \quad \mathrm{Ua}=0,2 \mathrm{~m} / \mathrm{s}$ & 1,6 & 1,6 & 0 & $\mathbf{0 , 0 0 \%}$ \\
\hline $\mathrm{D} 50=90 \mu \mathrm{m} \quad \mathrm{Ua}=0,25 \mathrm{~m} / \mathrm{s}$ & 2,2 & 2 & 0,2 & $\mathbf{9 , 0 9 \%}$ \\
\hline
\end{tabular}

\begin{tabular}{|c|c|c|c|c|}
\hline $100 \%$ sable 4,5 litres Ua $=0 \mathrm{~m} / \mathrm{s}$ & $\mathrm{T}(\mathrm{s})$ chute & $\mathrm{T}(\mathrm{s})$ chute & Erreur & Erreur \\
\hline $\mathrm{D} 50=90 \mu \mathrm{m}$ Influence $\boldsymbol{d e} \boldsymbol{C}$ & essai & bi-espèce $2 \mathrm{D}$ & $\Delta \mathrm{t}(\mathrm{s})$ & $\%$ \\
\hline $\mathrm{C}=100 \mathrm{~g} / 1$ & 3,4 & 3,5 & 0,1 & $\mathbf{2 , 9 4 \%}$ \\
\hline $\mathrm{C}=100 \mathrm{~g} / 1$ & 3,8 & 3,5 & 0,3 & $\mathbf{7 , 8 9 \%}$ \\
\hline $\mathrm{C}=100 \mathrm{~g} / 1$ & 3,7 & 3,5 & 0,2 & $\mathbf{5 , 4 0 \%}$ \\
\hline $\mathrm{C}=400 \mathrm{~g} / 1$ & 1,6 & 1,7 & 0,1 & $\mathbf{6 , 2 5 \%}$ \\
\hline $\mathrm{C}=200 \mathrm{~g} / 1$ & 2,47 & 2,6 & 0,13 & $\mathbf{5 , 2 6 \%}$ \\
\hline $\mathrm{C}=200 \mathrm{~g} / 1$ & 2,53 & 2,6 & 0,07 & $\mathbf{2 , 7 6 \%}$ \\
\hline
\end{tabular}

\begin{tabular}{|c|c|c|c|c|}
\hline Sablo vaseux 45 litres $350 \mathrm{~g} / \mathrm{l}$ & $\mathrm{T}(\mathrm{s})$ chute & $\mathrm{T}(\mathrm{s})$ chute & Erreur & Erreur \\
\hline $\mathrm{D} 50($ sable $)=90 \mu \mathrm{m}$ D $50($ vase $)=15 \mu \mathrm{m}$ & & & & \\
\hline Influence \% sable et courant & essai & bi-espèce $2 \mathrm{D}$ & $\Delta \mathrm{t}(\mathrm{s})$ & $\mathbf{\%}$ \\
\hline $20 \%$ sable Ua $=0 \mathrm{~m} / \mathrm{s}$ & 2,8 & 2,7 & 0,1 & $\mathbf{3 , 5 7 \%}$ \\
\hline $60 \%$ sable $\mathrm{Ua}=0 \mathrm{~m} / \mathrm{s}$ & 2,52 & 2,5 & 0,02 & $\mathbf{0 , 7 9 \%}$ \\
\hline $20 \%$ sable Ua $=0,1 \mathrm{~m} / \mathrm{s}$ & 3,74 & 3,7 & 0,04 & $\mathbf{1 , 0 7 \%}$ \\
\hline $60 \%$ sable Ua $=0,1 \mathrm{~m} / \mathrm{s}$ & 3,53 & 3,5 & 0,03 & $\mathbf{0 , 8 5 \%}$ \\
\hline $100 \%$ sable $\mathrm{Ua}=0 \mathrm{~m} / \mathrm{s}$ & 1,76 & 1,8 & 0,04 & $\mathbf{2 , 2 7 \%}$ \\
\hline $100 \%$ sable $\mathrm{Ua}=0,1 \mathrm{~m} / \mathrm{s}$ & 2,02 & 2 & 0,02 & $\mathbf{0 , 9 9 \%}$ \\
\hline
\end{tabular}

Tableau 3. Comparaison des temps de chute de rejets $100 \%$ sable ou sablo vaseux avec ou sans courant ambiant

Ces différents essais sont exprimés selon trois groupes permettant l'étude de l'influence du courant ambiant sur les rejets $100 \%$ sable, l'étude de l'influence de la concentration initiale du rejet et enfin l'importance du pourcentage de sable et du courant ambiant sur les rejets sablo vaseux.

En adéquation avec les observations expérimentales, nous retrouvons par ailleurs dans les résultats du modèle, l'augmentation sensible du temps de chute avec l'intensité du courant ambiant et ce pour tous les types de rejets.

De même, le modèle subit correctement l'influence de la concentration initiale avec une nette diminution du temps de chute au fil de l'augmentation de la concentration sédimentaire. Enfin, nous observons bien également une augmentation sensible du temps de chute avec la diminution du pourcentage de sable dans les rejets sablo vaseux. 
La figure 6 présente la comparaison de trois simulations de 45 litres de rejets $100 \%$ vase de diamètre $10 \mu \mathrm{m}$ et de concentration volumique initiale fixée à $350 \mathrm{~g} / \mathrm{l}$. Clap 15, Clap 16 et Clap 17 sont les noms donnés aux essais physiques (FaroutFréson, 2004) correspondant à des courants ambiants respectifs de $10,6 \mathrm{~cm} / \mathrm{s}, 5,2 \mathrm{~cm} / \mathrm{s}$ et $20,1 \mathrm{~cm} / \mathrm{s}$. La « relative irrégularité » des courbes est due à la technique localisation du front d'après un seuil de concentration donné et l'espacement des mailles qui font que le front est toujours déterminé à une demi-longueur d'éléments près.

Malgré le fait que le modèle bi-espèce 2D ne tienne pas compte de la rhéologie spécifique des sédiments cohésifs, l'influence du courant ambiant sur la phase de chute est correctement reproduite et nous assistons bien à une augmentation du temps de chute et du rayon du rejet à l'impact avec l'augmentation du courant ambiant au sein du canal.

Sur l'ensemble des simulations réalisées, la variation totale de la masse sédimentaire au cours du temps est restée inférieure à $0,2 \%$ quelle que soit le type de rejet envisagé. Les rejets sans courant ambiant sont cependant plus conservatifs avec une perte de masse comprise entre $0,0194 \%$ et $0,1342 \%$ alors que les rejets avec courant accusent une perte comprise entre $0,1570 \%$ et $0,1780 \%$.
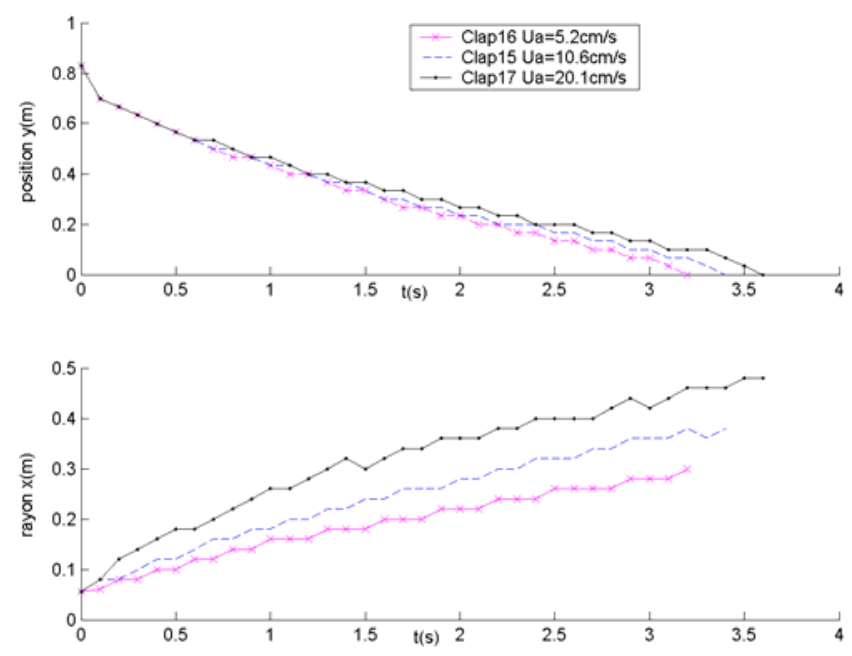

Figure 6. Influence du courant ambiant sur la phase de chute de rejet $100 \%$ vase

Les résultats du modèle sur la phase de chute de différents types de rejets de dragage par clapage montrent que les différentes positions du nuage obtenues par le modèle bi-espèce $2 \mathrm{D}$ sont en quasi-adéquation avec les données expérimentales. Les temps de chute numériques sont en accord avec les temps de chute expérimentaux dans la totalité des simulations effectuées et les influences du courant ambiant, de la concentration initiale ou du pourcentage de sable dans les rejets sablo vaseux sont 
respectées. La vitesse de chute numérique proposée donne un caractère opérationnel au modèle, étant exclusivement définie en fonction des données initiales du rejet considéré. Cette nouvelle approche de la modélisation de la vitesse de chute sédimentaire permet ici une bonne reproduction du comportement du rejet qu'il soit $100 \%$ sable ou sablo vaseux dans un milieu avec ou sans courant ambiant. L'influence du courant ambiant est parfaitement reproduite pour les rejets de type $100 \%$ vase malgré le fait que ce modèle ne tienne pas compte de la rhéologie des sédiments cohésifs. Dans son ensemble le modèle présenté est cohérent pour toute la phase de chute de tous les types de clapage. Ce modèle permet aussi d'approcher les deux autres phases du clapage avec l'impact au sol et le transport des sédiments par courant de densité et les premiers résultats numériques obtenus (intensité du courant de densité et concentration de sédiment transportée sur le fond) sont encourageants : la figure 7 donne la fraction massique maximum de sédiment transporté par courant de densité en fonction de l'avancée du rejet sur le fond pour un rejet $100 \%$ sable sans courant (la valeur OPCON expérimentale est de $13 \mathrm{~g} / \mathrm{l}$ à $1 \mathrm{~m}$ de l'axe du rejet contre une valeur de $11.40 \mathrm{~g} / \mathrm{l}$ pour le modèle bi-espèce).

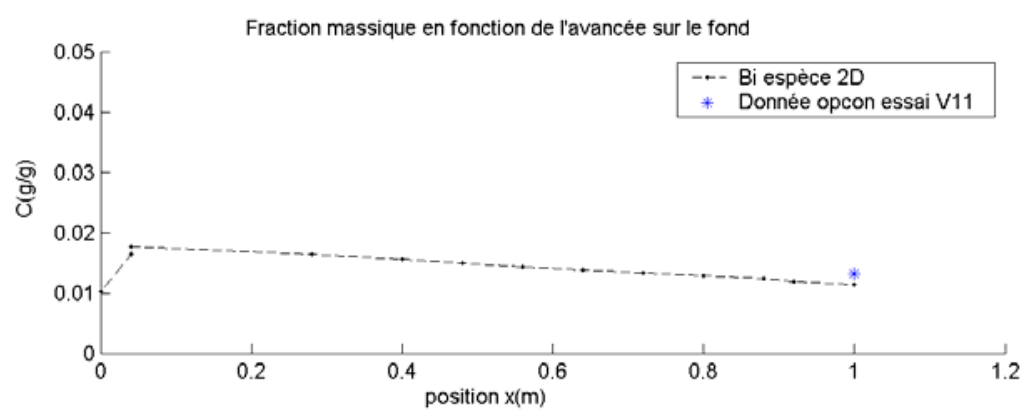

Figure 7. Phase de transport d'un rejet $100 \%$ sable sans courant ambiant: fraction massique de sédiment transportée sur le fond

\section{Remerciements}

L'auteur principal tient ici à remercier la Région Picardie pour avoir financé ce travail de novembre 2000 à juin 2003.

\section{Bibliographie}

Anderson T.B., Jackson J., "A fluid mechanical description of fluidized beds", Ind. Eng. Chemical Fundamental, vol. 6, n 4, 1967, p. 527-539. 
Bokuniewicz H.J., Gebert J., Gordon R.B., Higgins J.L., Kaminsky P., Pilbeam C.C., Reed M., Tuttle C., Field study of the mechanics of the placement of dredged material at open water disposal site, New Haven, Connecticut (USA), Yale University for USAEWES, TR D-78-7, 1978.

Bokuniewicz H.J., Gordon R.B., "Deposition of dredged sediment at open water sites", Estuarine and coastal marine science, 10, 1980, p. 289-303.

Boutin R., Amélioration des connaissances sur le comportement des rejets en mer de produits de dragage de type vase, phénomènes à court terme et dans le champ proche, Thèse de doctorat de l'Institut National des Sciences Appliquées de Lyon, 1999.

Burel D., Villaret C., Modélisation de la dispersion des rejets par clapage projet LITEAU, Rapport EDF HP-72/2000/054/A 2000.

Dhatt G., Hubert G., "Some new penalty elements for incompressible flows", $3^{\text {th }}$ Int. Conf. On Num. Meth., Laminar and turbulent flows, 1983, Seatle - USA.

Dhatt G., Touzot G., Lefrançois E., Méthode des éléments finis, Hermès, 2005.

Drapeau G., Lavallee D., Dumais J.F., Walsh G., "Dispersion model of dredge spoil dumped in coastal water", Coastal engineering, Venice Italy 4-9 October 1992, p. 3054-3067.

Farout-Fréson I., Modélisation du clapage en mer ; étude du champ proche ; chute et transport sur le fond, Thèse de doctorat de l'Université de Technologie de Compiègne spécialité mécanique : hydraulique numérique, 2004.

Farout-Fréson I., Lefrançois E., Dhatt G., Sergent P., "Finite element model of dredged material dumped into sea: comparison with flume experiments", $3^{\text {rd }}$ IAHR Symposium on River, Coastal and Estuarine Morphodynamics, Barcelona 2003, p. 1187-1195.

Farout-Fréson I., Lefrançois E., Dhatt G., Sergent P., « Modèle numérique bi-espèce 2D pour les écoulements à densité variable fluide/particules: application au clapage », $10^{e}$ journées de l'hydrodynamique, Nantes 7-9 mars 2005, p. 23-36.

Gordon R.B., "Dispersion of dredged spoil dumped in near-shore waters", Estuarine and coastal marine science, tome 2, 1974, p. 349-358.

Jonhson B., Fong M., Development and verification of numerical models for predicting the initial fate of dredged material disposed in open water, Report 2, Theoretical developments and verification results, final report prepared for US army corps of engineers 20314-1000, February 1995.

Krishnappan B.G., "Dispersion of granular material dumped in deep water", Burlington, Ontario: environment Canada 113 pages scientific series $n^{\circ}$ 55, 1976.

Migniot C., «Etude des propriétés physiques de différents sédiments très fins et de leur comportement sous des actions hydrodynamique », La Houille Blanche, n 7, 1989, p. 591-619.

Piperno S., Farhat C., "Energy based design and analysis of staggered solvers for nonlinear transient aeroelastic problems", AIAA Paper, 2000, p. 2000-1447.

Richardson Y.F., Zaki W.N., "Sedimentation and fluidization: part I", Trans. Inst. Chem. Engineering, vol. 32, 1954, p. 35-53. 
988 Revue européenne de mécanique numérique. Volume 16 - n 8/2007

Rouas G., Etude et modélisation par éléments finis des processus hydro sédimentaires estuariens, Thèse de doctorat en sciences mécaniques pour l'ingénieur à l'Université de Technologie de Compiègne, 1996.

STFATE, http://el.erdc.usace.army.mil/dots/models.html (US Army Corps of Engineers).

Tambo N., Watanabe Y., Physical characteristics of flocs I: The floc density function and aluminium floc, Water Research, vol. 13, 1979, p. 409-419.

Truitt C.L., "Dredged material behaviour during open water disposal", Journal Coastal Research, vol. 4, n 3, p. 389-397, 1988.

Van Leussen W., "Aggregation of particles, settling velocity of fluid muds", Physical Processes in Estuaries, Edition Job Dronkers\&Wim Van Leussen, Springer Verlag, 1988, p. 347-403.

Van Leussen W., "Field measurements of flocs sizes and settling velocities", MAST G8M, Workshop Leuven, 5-6 April 1993.

Van Rijn L.C., "Principles of sediment transport in rivers, estuaries and coastal seas", Aqua publications, 1993, p. 3-13.

Villaret C., Lekien M., Claude B., Vinet F., Etude expérimentale de la dispersion des rejets par clapage d'un mélange de sable et de vase (influence du courant et de la concentration), Rapport EDF HE-42/97/072/A 1997. 